\title{
915 nm Pumped 1018 nm Yb-Doped All-Fiber High Power Fiber Laser System
}

\author{
Yakup Midillii ${ }^{1}$, O. Benjamin Efunbajo ${ }^{1,2}$, Bartu Şimşek $^{1}$ and Bülend Ortaç ${ }^{1}$ \\ 1. UNAM - National Nanotechnology Research Center and Institute of Materials Science and Nanotechnology, Bilkent University, 06800, Bilkent, \\ Ankara, Turkey \\ 2. Present address: Department of Health Technology, Technical University of Denmark, Anker Engelunds Vej 1, Building 101A, 2800 Kgs. Lyngby, \\ Denmark
}

Fibers lasers have attracted great attention in the last decades and the power scaling has reached tens of $\mathrm{kW}$ levels. Especially with the tandem pump configuration, pumping the active medium with a pump laser light instead of a diode laser, has made a breakthrough and so many research has been conducted about $1018 \mathrm{~nm}$ fiber laser systems [1]. Decreasing the quantum defect, the slope efficiency could be increased up to $90 \%$ levels; on the other hand, due to the emission cross section of the Ytterbium (Yb), to operate the laser in the $1018 \mathrm{~nm}$ wavelength region is very challenging because of the presence of the ASE about the $1030 \mathrm{~nm}$ region. However, in the literature by using $976 \mathrm{~nm}$ pump diodes multi-hundred watts level $1018 \mathrm{~nm}$ fiber lasers could be demonstrated [2, 3].

In this work, we have developed a $1018 \mathrm{~nm}$ high power fiber laser by pumping with a $915 \mathrm{~nm}$ pump diode instead of $976 \mathrm{~nm}$ pump diode. Additionally, by changing the wavelength of the pump source from $904 \mathrm{~nm}$ to 924 $\mathrm{nm}$ with a $20 \mathrm{~nm}$ span we have showed the laser action continue with a stable efficiency.

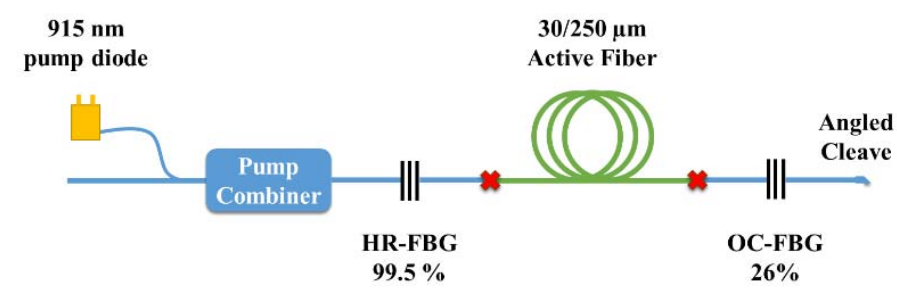

Fig. 1 Schematic illustration of $1018 \mathrm{~nm}$ fiber laser system.

We have established a setup which includes a $915 \mathrm{~nm}$ high power pump diode combined to a pump combiner and a pair of FBGs working at $1018 \mathrm{~nm}$ wavelength and an active fiber having core/cladding diameters $30 / 250 \mu \mathrm{m}$ respectively as shown in Fig.1.
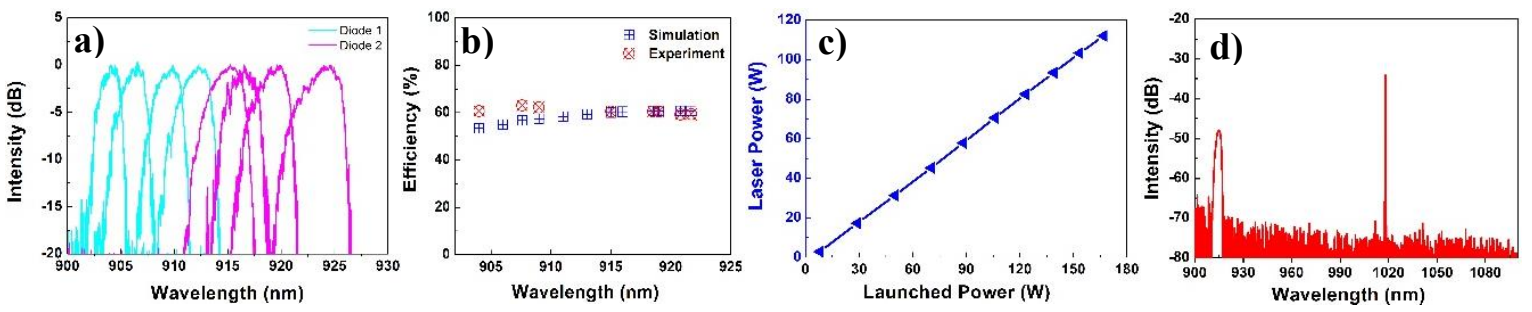

Fig. 2 Optical spectrum characterization of two different pump sources with the help of a heater (a), the efficiency change with respect to the change in the pump wavelength (b), power characterization of the $1018 \mathrm{~nm}$ fiber laser (c) and the optical spectrum at its maximum power $(\mathrm{d})$.

Firstly, we have characterized the central wavelength change of pump diodes by increasing their temperatures as in the Fig. 2(a) and tested the change in the laser efficiency and observed that the experimental results coincide with the simulation performed by RP-Fiber Power software as in the Fig. 2(b). Finally, we have tested the high power performance of the laser system and obtained a power above $100 \mathrm{~W}$ as shown in Fig. 2(c) and the corresponding optical spectrum at its maximum power shown in Fig. 2(d). Therefore, we have succeeded to demonstrate the first that $1018 \mathrm{~nm}$ fiber laser systems could be pumped by $915 \mathrm{~nm}$ pump wavelength region as well. As a result, benefiting from the broad absorption region of $\mathrm{Yb}$ atoms around $915 \mathrm{~nm}$ wavelength region we have also demonstrated that stable $1018 \mathrm{~nm}$ fiber laser systems could be developed.

\section{References}

[1] H. Xiao, J. Leng, H. Zhang, L. Huang, J. Xu, and P. Zhou, "High-power $1018 \mathrm{~nm}$ ytterbium-doped fiber laser and its application in tandem pump," Applied Optics 54, 8166 (2015).

[2] C. Ottenhues, T. Theeg, K. Hausmann, M.Wysmolek, H. Sayinc, J. Neumann, and D. Kracht, "Single-mode monolithic fiber laser with 200 W output power at a wavelength of $1018 \mathrm{~nm}$," Opt. Lett. 40, 4851 (2015).

[3] Y. Glick, Y. Sintov, R. Zuitlin, S. Pearl, Y. Shamir, R. Feldman, Z. Horvitz, and N. Shafir, "Single-mode $230 \mathrm{~W}$ output power $1018 \mathrm{~nm}$ fiber laser and ASE competition suppression,” J. Opt. Soc. Am. B 33, 1392 (2016). 